\title{
Major abdominal wall defects in the low- and middle-income setting: current status and priorities
}

\author{
Lofty-John Chukwuemeka Anyanwu ${ }^{1} \cdot$ Niyi Ade-Ajayi ${ }^{2} \cdot$ Udo Rolle $^{3}$
}

Accepted: 5 March 2020 / Published online: 21 March 2020

(c) The Author(s) 2020

\begin{abstract}
Major congenital abdominal wall defects (gastroschisis and omphalocele) may account for up to $21 \%$ of emergency neonatal interventions in low- and middle-income countries. In many low- and middle-income countries, the reported mortality of these malformations is $30-100 \%$, while in high-income countries, mortality in infants with major abdominal wall reaches less than 5\%. This review highlights the challenges faced in the management of newborns with major congenital abdominal wall defects in the resource-limited setting. Current high-income country best practice is assessed and opportunities for appropriate priority setting and collaborations to improve outcomes are discussed.
\end{abstract}

Keywords Abdominal wall defects · Gastroschisis · Omphalocele $\cdot$ Low- and middle-income countries $\cdot$ High-income countries

\section{Introduction}

Congenital anomalies account for $10 \%$ of global neonatal deaths, with children in low- and middle-income countries (LMICs) being disproportionately affected [1,2]. Major congenital abdominal wall defects (gastroschisis and omphalocele ) may account for up to $21 \%$ of emergency neonatal interventions in the LMIC setting [3, 4]. If infants with these conditions present with associated involvement of major organ systems, their management may be complicated $[2,5]$.

Gastroschisis (GS) and omphalocele (particularly with ruptured sac) are associated with fluid shifts and physiological alterations that make management challenging in the resource-constrained setting. This may be exacerbated by suboptimal neonatal transportation to the appropriate health facility. In many LMICs, the reported mortality is $30-100 \%$

Udo Rolle

udo.rolle@kgu.de

1 Paediatric Surgery Unit, Department of Surgery, Aminu Kano Teaching Hospital and Bayero University, Kano, Nigeria

2 Department of Paediatric Surgery, King's College Hospital, London, UK

3 Department of Paediatric Surgery and Paediatric Urology, University Hospital Frankfurt/M., Theodor-Stern-Kai 7, 60598 Frankfurt, Germany
[6-8], while in high-income countries (HICs), mortality in infants with major abdominal wall defects is less than $5 \%$ $[9,10]$.

This review highlights the challenges faced in the management of newborns with major congenital abdominal wall defects in the resource-limited setting.

Current HIC best practice is assessed and opportunities for appropriate priority setting and collaborations to improve outcomes are discussed.

\section{Gastroschisis}

\section{Aetiopathogenesis}

GS is a full-thickness congenital abdominal wall defect typically located to the right of the umbilicus. Through this defect, small and large bowel and sometimes other intraabdominal viscera herniate into the amniotic cavity and are bathed by amniotic fluid in utero. Historically, GS was reported to have an incidence of 1 in 4000 live births. However, recent data suggest that there have been substantial increases in incidence globally over the past two decades [11-14].

It is believed that GS results from in utero involution of the right umbilical vein, which results in necrosis and a full-thickness defect in the right paraumbilical area [11, 
Table 1 Selected variables of patients with gastroschisis (AKTH Kano)

\begin{tabular}{llll}
\hline Parameter & Minimum & Maximum & Median \\
\hline Age on admission in days & 1 & 6 & 2 \\
Gestational age at birth in weeks & 33 & 40 & 38 \\
Age of mother in years & 15 & 35 & 19 \\
Age of father in years & 25 & 45 & 30 \\
Weight on admission in kg & 1.30 & 3.20 & 2.20 \\
Age in days at time of first surgery & 1 & 10 & 3 \\
Age in days at time of second & 3 & 13 & 7.5 \\
$\quad$ surgery & & & \\
Age in days at full oral feeds & 3 & 18 & 9.5 \\
Age in days at time of death & 1 & 26 & 3 \\
Length of hospital stay in days & 1 & 26 & 3 \\
\hline
\end{tabular}

Table 2 Associated anomalies (AKTH gastroschisis study)

\begin{tabular}{llc}
\hline Anomaly & Number $(n)$ & $\%(n / 39)$ \\
\hline Intestinal atresia & 1 & 2.6 \\
Polydactyly & 1 & 2.6 \\
Talipes equinovarus & 1 & 2.6 \\
Bowel ischaemia/gangrene & 4 & 10.26 \\
Pansystolic murmur & 1 & 2.6 \\
Total & 8 & 20.66 \\
\hline
\end{tabular}

$14,15]$. Others have suggested that early involution of the right vitelline (omphalomesenteric) artery causes a paraumbilical abdominal wall defect $[14,15]$. Although the exact cause of GS is unknown, young maternal age and interactions between gene polymorphism, cigarette smoking, and illicit drug use are suggested predisposing factors $[16,19]$.

In a retrospective review of 39 patients managed for gastroschisis at the Aminu Kano Teaching Hospital Kano (AKTH), Nigeria (Table 1), over a 5-year period, the median maternal age was 19 years (range 15-35).

In GS, the determinants of intestinal damage include the duration of exposure to amniotic fluid and the size of the abdominal wall defect $[12,17]$. As the pregnancy advances, pressure from the closing abdominal wall defect may result in venous and lymphatic obstruction, which is deleterious to the bowel. In addition, the inflammatory effect of the amniotic fluid on the bowel results in the development of a fibrinous peel, which thickens and mats the bowel, with resultant reduction in the lumen and motility $[14,17]$.

Between 10 and $20 \%$ of patients with GS have an associated anomaly, the majority of which are in the gastrointestinal tract [11]. Some of the reported associations are intestinal atresia and duplications, volvulus, and Meckel's diverticulum $[11,15,20]$. Table 2 shows the associated anomalies seen in patients in the AKTH review of GS outcome.

\section{Prenatal diagnosis and care}

The earliest indicator of the presence of a foetus with gastroschisis may be elevation of maternal serum $\alpha$-fetoprotein (up to 9 multiples of the mean) $[11,14,15]$. Maternal abdominal sonography at 18 weeks helps to confirm the diagnosis [14]. Once GS is confirmed, other investigations may be appropriate; these may include foetal echocardiography and amniocentesis to exclude other anomalies [14, 15, 20]. Following antenatal diagnosis, delivery should be scheduled in a centre with a team of professionals that include a paediatric surgeon a and neonatologist $[14,15]$.

All (100\%) of the patients in the AKTH Kano review were "out-born" and none was prenatally diagnosed. Similar findings have been reported from the sub-region $[6,9]$, and other reports suggest that two-thirds of the deliveries in many LMICs are home births [1].

\section{Delivery and early postnatal care}

The best method, as well as optimum timing of delivery for infants with GS, is debatable [14]. Given that neonates with gastroschisis and ruptured omphalocele have exposed bowel, heat and fluid losses are a major challenge. Also, relative intestinal hypomotility makes them prone to vomiting and aspiration pneumonitis. Early management includes passage of a nasogastric (NG) tube to decompress the stomach and protect the airway, adequate fluid resuscitation, and bowel protection. In some units, the use of warm-saline soaked gauze is traditional although this in itself may promote heat loss after the warm saline cools. Nursing the neonate in a controlled thermal environment is important $[11,14,15,20$, 21]. To prevent further damage of the bowel by kinking of the mesenteric vessels prior to surgery, the baby is nursed in the right lateral position [14, 20]. Associated anomalies should intentionally be sought for.

Given that conventional central venous catheters are not available, newborns with GS in AKTH Kano undergo cannulation of the umbilical vein with a size $5 \mathrm{~F}$ feeding tube to provide secure central venous access. Typically, these last for about 5 days before occlusion or dislodgement. Peripheral venous access is then utilized, but, in practice, is a challenging option.

\section{Surgical closure of the defect}

Following fluid resuscitation and stabilization of the infant with GS, return of eviscerated bowel to the abdominal cavity is undertaken. This can be carried out as a primary or staged (delayed) procedure if the bowel is viable $[15,22]$. The ideal 
method for the reduction of eviscerated bowel is debatable; however, the condition of the herniated viscera and the level of the visceroabdominal disproportion play a role in informing the choice of technique [12, 15, 17, 21].

At AKTH Kano, primary reduction and closure is the usual choice if immediate closure will not result in excessive intraabdominal pressure or respiratory compromise $[15,17]$. Some authors recommend primary closure for all babies with gastroschisis and a favourable anatomy [23], while others have shown that primary fascial closure is not practicable in up to $79 \%$ of neonates with gastroschisis [24]. In the presence of a significant visceroabdominal disproportion, delayed fascial closure is done by the placement of either a preformed or a sutured silo, with serial tucks of the silo carried out on a daily basis until the herniated bowel is completely reduced. The use of the silo closure has been shown to be associated with better physiological parameters as well as patient outcomes [25]. Another key advantage of this strategy is the conversion of an emergency clinical situation into one that can be managed in a more measured way $[26,27]$.

A retrospective review of the outcome of patients managed with GS in AKTH Kano between September 2011 and May 2016 was carried out; of 39 patients, 26 (66.67\%) were girls and $13(33.33 \%)$ boys, giving a female to male ratio of

Table 3 First intervention on presentation (AKTH gastroschisis study)

\begin{tabular}{lll}
\hline Intervention & Number & $\%$ \\
\hline Improvised silo bags (sutured) & 11 & 28.2 \\
Improvised silo bag (non-sutured) & 9 & 23.1 \\
Primary closure & 6 & 15.4 \\
Died before surgical intervention & 13 & 33.3 \\
Total & 39 & 100 \\
\hline
\end{tabular}

2:1. Other characteristics of the study patients are displayed in Table 1. Our patients are usually admitted onto the special care baby unit (SCBU), as we do not have a neonatal intensive care unit (NICU). The patients are co-managed by neonatologists and paediatric surgeons with the surgical team assuming primary clinical responsibility. Following the initial fluid resuscitation and bowel covering, the patient is nursed in an incubator. Prior to October 2014, all patients underwent surgery under general anaesthesia (GA) in the operating theatre. Those patients, who had significant visceroabdominal disproportion and viable bowel with no intestinal atresias, had a sutured improvised silo placed under GA, using a sterile urine bag as silo material. Those patients, who had complex GS with gangrene or intestinal atresia, underwent resection of the pathologic bowel segment, a primary intestinal anastomosis, and primary abdominal wall (fascia and skin) closure (Table 3).

Evidenced by the poor outcomes of patients with GS, a decision was made to de-emphasize general anaesthesia in the management of those infants without intestinal pathology, as most died peri-operatively. From October 2014, this cohort has been managed with an improvised silo placed in SCBU under sedation with IV-diazepam $(0.1 \mathrm{mg} / \mathrm{kg}$ slow IV push). The silo is fashioned from a sterile urine bag and a rubber ring from an automobile oil filter (Fig. 1a-c). We sterilize the rubber ring by first washing with a detergent and soaking in activated dialdehyde solution $\left(\mathrm{Cidex}^{\mathrm{TM}}\right)$ for $30 \mathrm{~min}$. When this solution is not available, we soak in $10 \%$ povidone iodine for $30 \mathrm{~min}$. Recently, we started to autoclave the rings and preserve them in a sterile container. All our patients received intravenous antibiotics (ceftriaxone $100 \mathrm{mg} / \mathrm{kg} /$ day and metronidazole $7.5 \mathrm{mg} / \mathrm{kg} /$ dose 8 hourly) throughout the period of their admission. None of them was ventilated.

Following complete reduction of the bowel into the peritoneal cavity (Fig. 2), the abdominal wall is closed under
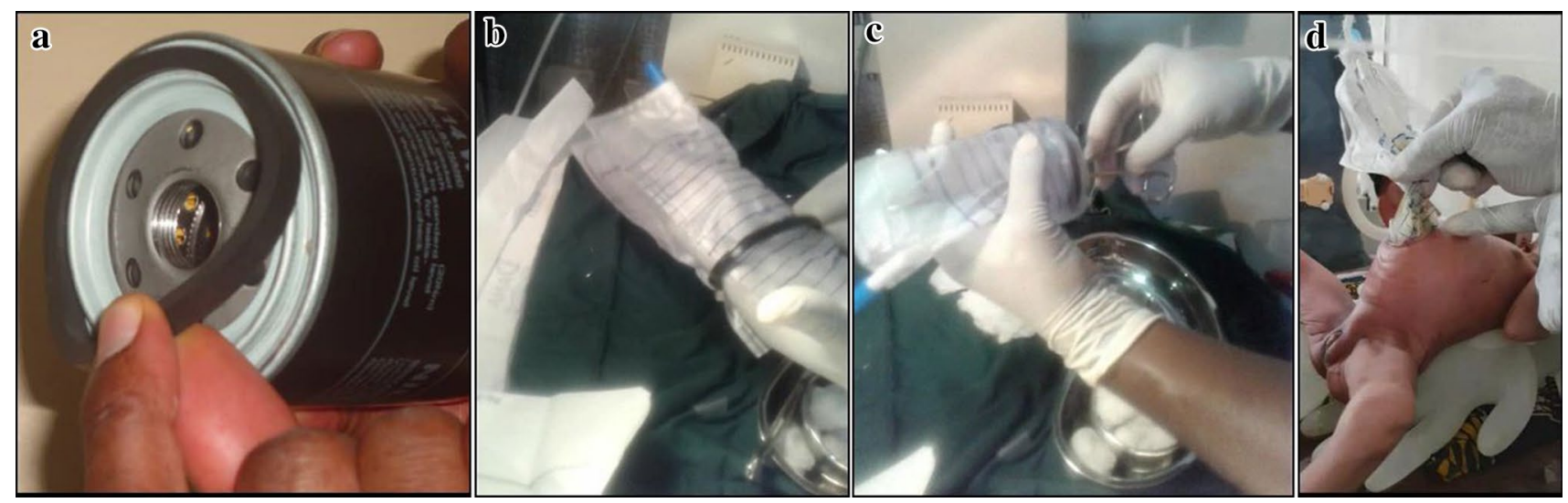

Fig. 1 a Rubber ring of automobile oil filter. b Cut end of urine bag fitted into the rubber ring. $\mathbf{c}$ Cut end of bag everted over the rubber ring and held in place by non-absorbable sutures to form the silo bag (Kano bag) 


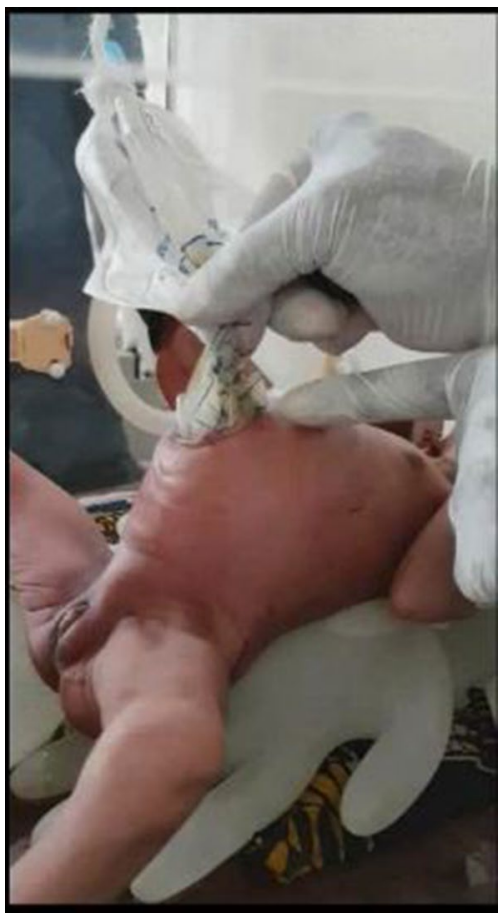

Fig. 2 Fashioned silo bag, clinical application and complete reduction of the bowel

local anaesthesia and sedation (muscle and fascia separate from skin) with non-absorbable sutures (Nylon 2/0) and dressed. The baby is returned to the incubator nil per os (NPO) with continuous nasogastric (NG) tube drainage and IV fluids. If by the second day post-closure, the NGtube aspirate has reduced significantly or the baby is passing stools, the baby is commenced on breast milk that is increased as tolerated.

\section{Treatment outcome}

Noting the high mortality rate in infants with gastroschisis who had a bowel pathology (bowel stenosis, bowel atresia, bowel ischaemia, or bowel perforation), Molik and colleagues categorized GS into two groups, i.e. simple (without intestinal pathology) and complex (with intestinal pathology), to aid the comparison of outcome between series and the categorization of risk, and this has been adopted by many $[9,21,28-30]$. Given that the causes of death in infants with GS are diverse [10,27,31], the capacity of a particular centre to address the various aspects of the compromised neonate with gastroschisis will impact on their outcome. Ford et al. had demonstrated that avertable DALYs (or unmet need) in the management of neonates with GS were lowest in HICs and highest in low-income countries (LICs). They concluded that GS outcomes for any particular unit
Table 4 Association between mortality and selected variables of the neonates with gastroschisis (AKTH study)

\begin{tabular}{|c|c|c|c|c|c|}
\hline \multirow[t]{2}{*}{ Variable } & \multirow[t]{2}{*}{$n$} & \multirow[t]{2}{*}{ Died } & \multirow[t]{2}{*}{ Alive } & \multicolumn{2}{|c|}{$\begin{array}{l}\text { Statistical signifi- } \\
\text { cance }\end{array}$} \\
\hline & & & & $\chi^{2}$ & $p$ value \\
\hline \multicolumn{6}{|l|}{ Sex } \\
\hline Male & 13 & 11 & 2 & 0.115 & 0.735 \\
\hline Female & 26 & 23 & 3 & & \\
\hline \multicolumn{6}{|c|}{ Sepsis on presentation } \\
\hline Yes & 21 & 20 & 1 & 2.644 & 0.162 \\
\hline No & 18 & 14 & 4 & & \\
\hline \multicolumn{6}{|c|}{ Molik's classification } \\
\hline Simple & 34 & 30 & 4 & 0.264 & 0.517 \\
\hline Complex & 5 & 4 & 1 & & \\
\hline \multicolumn{6}{|c|}{ Birth weight (kg) } \\
\hline$<2.5$ & 26 & 24 & 2 & 3.005 & 0.119 \\
\hline$\geq 2.5$ & 10 & 7 & 3 & & \\
\hline \multicolumn{6}{|c|}{ Gestational age (weeks) } \\
\hline$<37$ & 7 & 7 & 0 & 0.756 & 1.000 \\
\hline$\geq 37$ & 20 & 18 & 2 & & \\
\hline \multicolumn{6}{|c|}{ Age on admission (days) } \\
\hline 1 & 18 & 16 & 2 & 0.087 & 1.000 \\
\hline$>1$ & 21 & 18 & 3 & & \\
\hline
\end{tabular}

are a useful barometer of the capacity of that unit to deliver generic neonatal surgical care [10].

Mortality in the AKTH Kano series was 87.2\% (34/39). All the patients were born outside the hospital; 21 (53.8\%) were septic and all were hypothermic at presentation. Earlier studies from our sub-region had shown that septicaemia and hypothermia were common causes of death in infants with gastroschisis $[6,8,10]$. Analysis of certain patient-related variables in this series, however, did not show any that was significantly associated with mortality (Table 4). Late presentations and unaccounted for system-related variables may be responsible for the recorded deaths. Figure 3 shows the treatment algorithm for the management of gastroschisis in AKTH Kano.

It has been shown that the provision of parenteral nutrition to the neonate with GS during the prolonged period of post-operative ileus was critical to survival [21, 32]. Total parenteral nutrition is not available in our centre. To circumvent this, when we have several patients who require parenteral nutrition at the same time, their parents take turns to buy the daily amino acid infusions which can be shared among the neonates (a $200 \mathrm{ml}$ bottle costs about 14USD). We infuse this along with $10 \%$ dextrose infusions as 'partial parenteral nutrition'. The intralipid component is not easily available. It is usually difficult for one family to sustain this for a length of time. 
Fig. 3 The AKTH Kano gastroschisis algorithm

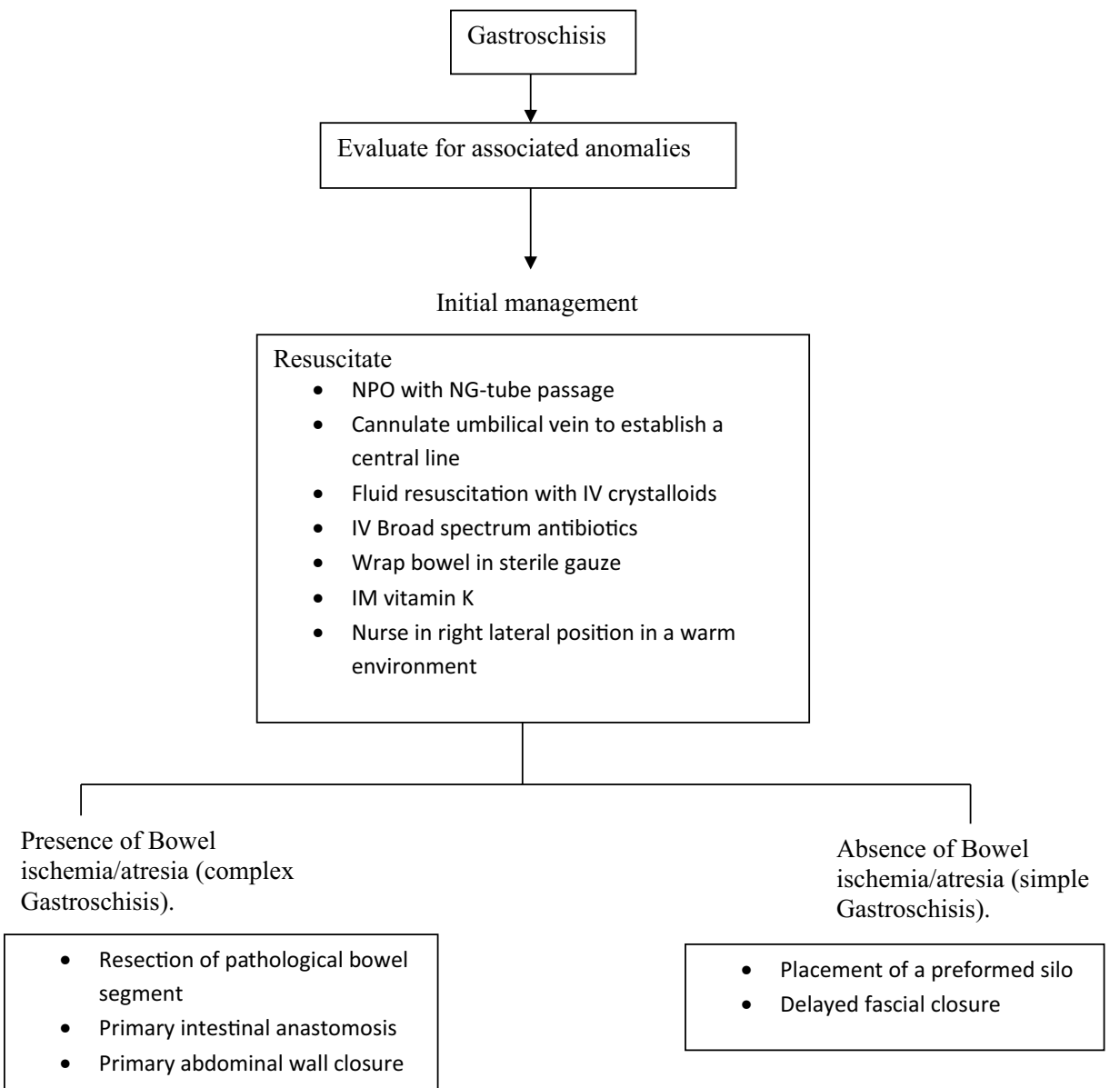

Prior to October 2014, we had no survivors of GS. The only survivors in this series were from the group of patients managed with the improvised sutureless silo. The consistent availability of total parenteral nutrition (TPN) may have resulted in additional survivors. Some of those who died in this group had reduction and abdominal wall closure, only to starve to death from the prolonged ileus. Analysis of our first ten cases managed with this method showed a $60 \%$ mortality rate, which was significantly different $(p=0.0351)$ from the outcome of our 11 patients managed with sutured silo under general anaesthesia.

\section{HIC lessons learnt and LMIC priorities for GS}

Over a period of 50 years, the outcomes of GS in HICs have been transformed. Allowing for resource constraints in LMICs, lessons can be learnt and outcomes improved in considerably shorter timeframes.

The HIC improvements have come from intentional planning and multi-disciplinary working at every phase of the journey of the mother, foetus, and then baby born with GS. These include the accurate confirmation of diagnosis during pregnancy, the monitoring of the pregnancy to pick up potential complications, and planned delivery at a centre with suitable expertise to manage the baby.

Standardized antenatal scans are taken for granted in the majority of HICs, but in many LMICs they are difficult to access, provision may be poorly regulated, and reliability is variable. In a recent series of ten infants born with GS in Kampala who underwent antenatal ultrasonography, only one was diagnosed [6]. Recommendation of antenatal scans as part of the standard package of care by the WHO would signal the importance of this aspect of obstetric care and could trigger the building of the relevant capacity to deliver it and contribute to improved outcomes for infants with GS among others. Regulation of training in public and private institutions would be important to develop and maintain standards.

Where there is a high proportion of out-born infants, developing networks of referral and care are an important contributor to the survival of infants who require intervention. This means that pre-hospital care, particularly resuscitation, bowel care, and principles of newborn transportation, can be standardized by local training and the use of protocols. 
Milestones in the improvement of GS outcomes in HICs included better recognition of the effects of abdominal compartment syndrome and the taking of steps to avoid and ameliorate these. These steps have included the use of improvised silos and, more recently, preformed silos to facilitate more gradual bowel reduction and the control of peritoneal fluid loss. These same technical strategies mean that reliance on general anaesthetic can be reduced, as this in itself may contribute to mortality in the sick, septic, hypothermic newborn.

While in HICs, the provision of consistent venous access is newborns with GS is given, in LMICs this can be very challenging. One of the solutions is highlighted in this report: the use of umbilical venous access to allow resuscitation, stabilization, and early nutrition. This can then be replaced in due course. Line care must be meticulous and infective complications closely monitored.

Over decades, HICs have built the capacity to deliver neonatal and paediatric intensive care. This provides the means to deal with those infants with a degree of abdominal compartment/respiratory compromise that is not available to colleagues at many LMIC settings. This situation could be improved with concerted national efforts and partnerships that will help make intensive care facilities more widely available in the LMIC setting.

The use of standardized parenteral nutrition in HICs has, unquestionably, provided the support to allow large numbers of infants to survive that hitherto would not have. However, infants in LMICs often do not survive the first few days of the condition. The data from Kampala [6] reflect death within the first 4-5 days of life in the majority of their patients. Therefore, while parenteral nutrition is a key component of modern-day care, early resuscitation and the avoidance of compartment syndrome appear to be even more important.

\section{Omphalocele}

\section{Aetiopathogenesis}

Omphalocele is a midline anterior body wall defect which is covered by a membrane. From inside outwards, the membrane covering the omphalocele defect consists of peritoneum, Wharton's jelly, and amnion [11, 14, 15, 20].

Usually, the umbilicus inserts into the membranous sac of the omphalocele defect [17]. Its incidence is $1-3$ per 10,000 live births, and appears to be stable so far $[11,18,33]$. The aetiology of omphalocele is not known with certainty; it is however believed to result from an abnormality of body wall infolding such that one or more of the folds (cephalic, caudal and two lateral) which close the ventral body wall at the umbilicus do not progress to the region $[5,11,17]$. Cephalic folding deficiencies result in an epigastric omphalocele commonly seen in pentalogy of Cantrell [11, 17, 34]. Lateral folding defects give rise to the commonly seen mid-abdominal omphalocele [11, 17], while deficiencies of infolding involving the caudal fold give a hypogastric omphalocele as in patients with cloacal or bladder exstrophy $[5,11,17]$.

Children with omphalocele have $>50 \%$ risk of having an associated anomaly, and about $50 \%$ of these have a genetic anomaly such as trisomy $13,14,15,18$ and 21 , and about $30-50 \%$ have a cardiac anomaly $[11,15,33,35]$. Beckwith Wiedemann syndrome (omphalocele, macroglossia, and gigantism) is said to occur in about $10 \%$ of infants with an omphalocele $[11,35]$. Infants with an omphalocele are believed to have a greater mortality risk than those with gastroschisis, because of their associated anomalies [18, 19, 33, 35]. Table 5 shows the associated overt anomalies seen in patients with omphalocele in AKTH Kano (Sept. 2011-February 2017).

\section{Prenatal diagnosis and care}

The use of antenatal ultrasonography and maternal serum $\alpha$-fetoprotein usually detects about $80 \%$ of ventral body wall defects $[11,15,17]$. Elevation of maternal serum $\alpha$-fetoprotein in omphalocele is less than that seen in gastroschisis, i.e. about four multiples of the mean $[11,15]$.

In HICs, routine prenatal ultrasound will be performed on the basis of structured protocols to discover major abnormalities. Prenatal ultrasound is very much sensitive for identifying omphalocele. Therefore, the prenatal detection rate of omphalocele is high, between 83 and $99 \%$, depending on the expression of the underlying disease, i.e. $83 \%$ for isolated cases, $95 \%$ of non-isolated, and $99 \%$ of chromosomal cases [36].

Most cases of omphalocele will be discovered by prenatal ultrasound at late first or early second trimester and result in high rates of termination and/or foetal loss (stillbirths). It has been estimated that around 30-49\% of omphaloceles present with chromosomal abnormalities (trisomy 13, 18, 21) and the majority $(85 \%)$ of these pregnancies will be terminated

Table 5 Associated anomalies (AKTH omphalocele study)

\begin{tabular}{lll}
\hline Anomaly & Number $(n)$ & $\%(n / 40)$ \\
\hline Beckwith Wiedemann syndrome & 5 & 12.5 \\
Myelomeningocele & 2 & 5 \\
Bowel gangrene & 1 & 2.5 \\
Cloacal exstrophy & 1 & 2.5 \\
Congenital heart disease & 1 & 2.5 \\
Down's syndrome & 1 & 2.5 \\
Parasitic twin & 1 & 2.5 \\
Anorectal malformation & 1 & 2.5 \\
Total & 13 & 32.5 \\
\hline
\end{tabular}


$[14,37]$. Furthermore, major associated anomalies in prenatally detected cases of omphalocele usually will lead to a decision of termination of pregnancy. Several reports confirm a $30-61 \%$ termination rate in prenatally diagnosed omphalocele [38].

Another interesting observation is that associated anomalies appear to be more common with minor omphaloceles $(2-5 \mathrm{~cm})$ than giant omphaloceles $(55 \%$ vs. $36 \%)$ [39]. A prenatally confirmed diagnosis of omphalocele should ultimately lead to the intrauterine transfer to a designated tertiary centre. This is to enable the decision making with regard to termination of the pregnancy or a structured pre-, peri-, and postnatal follow-up of these cases [40].

Pre- and perinatal mortality is high in omphalocele. A large cohort study revealed that $37 \%$ of the cases had termination of pregnancy or stillbirth, an additionally $15 \%$ died within the first 15 days after delivery, and that prenatally diagnosed and non-isolated omphaloceles had a significant higher mortality than postnatally diagnosed and isolated cases [41].

Delivery of prenatally diagnosed omphalocele patients is usually at term. The mode of delivery is under debate also in HICs, but should be mainly related to obstetric considerations. In fact, delivery mode is influenced by the type/size of omphalocele in some HICs (Table 6) [39, 42].

\section{Early postnatal care}

Like gastroschisis patients, infants with omphalocele are prone to increased fluid and heat loss. In neonates with a ruptured omphalocele, the heat and fluid loss are similar to that for infants with gastroschisis. The initial post-delivery management also consists of airway control and passage of a nasogastric (NG) tube, fluid resuscitation, temperature regulation (nurse child in a thermoneutral environment), and careful inspection of the sac to make sure it is still intact. The sac is subsequently covered in a warm saline-soaked gauze, after the child has been carefully evaluated for any associated congenital anomalies [15, 20,43].

Surgery of omphalocele is not an emergency procedure as long as the amniotic sac remains intact. Newborns should be placed in a sterile plastic bag (Fig. 4) to avoid fluid and temperature loss, which is less than in gastroschisis, but of course much higher when compared to newborns with an intact abdominal wall. Adequate resuscitation of the

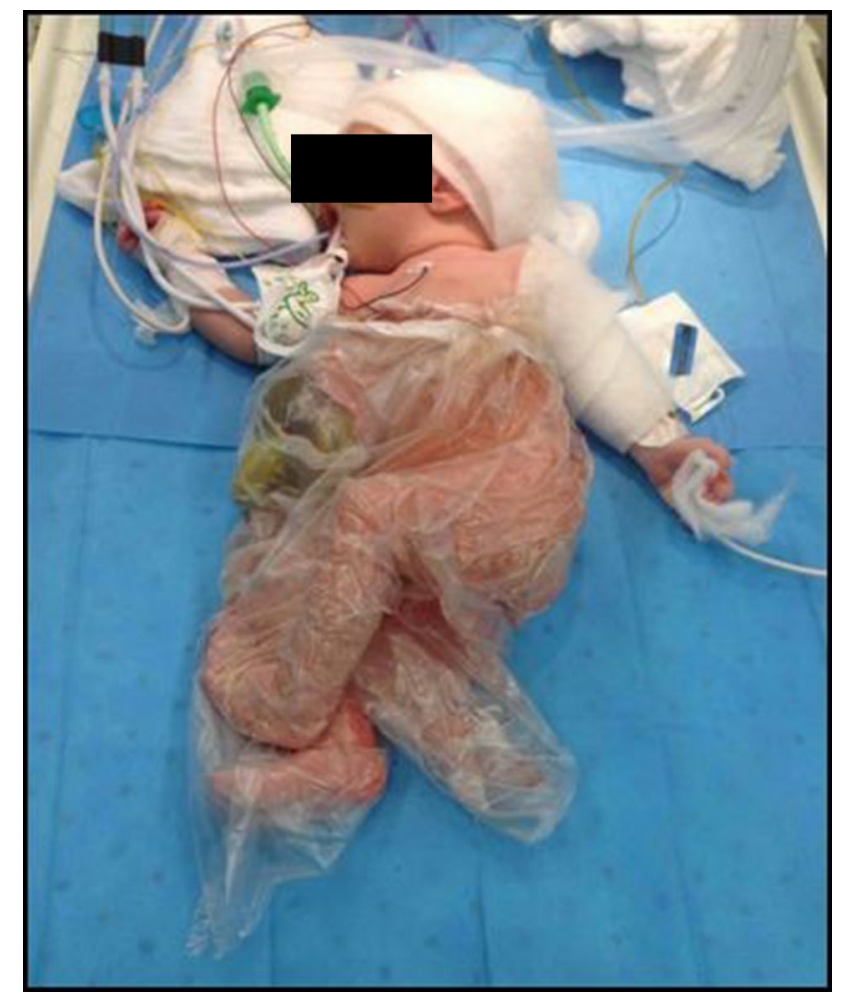

Fig. 4 Plastic bag for primary care

newborn and thorough assessment for additional congenital anomalies are necessary. First, the cardiopulmonary system of the newborn with omphalocele requires careful investigation, including echocardiography [44].

Concomitant pulmonary hypoplasia in giant omphalocele might require early intubation and ventilation. These cases would have been ideally already detected prenatally and would thus be known to the neonatology team. Naso- or orogastric tube is recommended for decompression of the gastrointestinal tract. An appropriate intravenous access should be secured for proper fluid resuscitation and for later surgery. If the newborn presents with a ruptured omphalocele, the treatment regime as in gastroschisis will be applied [45].

\section{Surgical closure of the defect}

The definitive treatment of an omphalocele depends on a number of considerations such as the integrity of the sac, the
Table 6 Type/size of defect, usual content, and modality of birth

\begin{tabular}{lll}
\hline Size of defect & Content of sac & Delivery \\
\hline Hernia to the cord & Small bowel, omphalomesenteric duct remnants & Vaginal at term \\
Minor defect $(2-5 \mathrm{~cm})$ & Small bowel/liver/stomach & Vaginal/caesarean at term \\
Large defect $(>5 \mathrm{~cm})$ & Liver, spleen, stomach, small bowel & Caesarean at term \\
\hline
\end{tabular}

Adopted from Gamba and Midrio [42], Verla et al. [39] 
size of the defect, the presence of associated anomalies, and the gestational age of the child $[11,14,15]$. As in the management of gastroschisis, the optimal approach to abdominal wall closure is debatable [11]. Depending on the size of the fascial defect, it may be classified as omphalocele major ( $\geq 5 \mathrm{~cm}$ of fascial defect) or omphalocele minor $(<5 \mathrm{~cm}$ of fascial defect) [15, 46, 47]. Some have used the term "giant omphalocele" to describe omphaloceles with the size of the fascial defect greater than $5 \mathrm{~cm}$ and which has the liver as one of the herniated viscera in the sac [48].

\section{Primary closure}

Primary closure, with excision of the amniotic sac and closure in layers (fascia and skin), is standard in small defects $(2-5 \mathrm{~cm})$, but could also be performed in large defects (Fig. 5).

There is no general consensus on the size of the defect and the content of the sac with regard to an attempt at primary closure. Single stage, early closure could be achieved in giant omphaloceles using either the anatomical closure in layers or the insertion of a synthetic patch into the fascia followed by skin closure [49].

Staged closure Gradual reduction using the amniotic sac itself and sequential ligation would be one of the options for staged closure. Staged closure using a synthetic prosthesis constructed like a silicon chimney is a useful method in giant omphaloceles [50].

Delayed closure Escharotic therapy can be done in selected cases, especially when primary closure is not feasible. Silver sulfadiazine is a commonly used escharotics agent. Patients could be kept in the ward and do not need intensive care treatment. Additionally, feeding is well tolerated during this treatment option. Treatment could be continued at home, including compressive dressing of the sac. Closure of the resulting abdominal hernia could be scheduled at the age of 6-12 months.

A systematic review of the methods of staged surgical vs. non-operative delayed closure revealed differences in mortality, length to full enteral feeding and length of hospital stay with a slight benefit seen in the delayed closure group. However, the authors did not take the necessary delayed closure surgery for the resulting ventral hernia in the nonoperative closure group into account [51].

A retrospective analysis of patients managed for an omphalocele in AKTH Kano between September 2011 and February 2017 showed that of the 40 patients whose records were analysed, 24 were males and 16 were females $(\mathrm{M}: \mathrm{F}=1.5: 1)$. Only one of the patients (2.5\%) had an antenatal ultrasound scan detection of the anomaly. Of the 40 patients, 27 (67.5\%) were born at home, while $13(32.5 \%)$ were born in a health facility. There were two (5\%) caesarean deliveries due to foetal distress. The majority $(35 / 40 ; 87.5 \%)$ of the neonates had an omphalocele major. Six patients presented with a ruptured sac. These six patients had an operative treatment, with the placement of a sutured silo bag under general anaesthesia. All other patients with an intact sac had a non-operative treatment with the use of an escharotic agent (silver sulphadiazine cream) topically applied to the sac to induce epithelialization and create a ventral hernia (Fig. 6) which would be closed at a later date [15, 43, 47, 48, 52, 53]. Other parameters of the study patients are as depicted in Table 7.
Fig. 5 Primary closure of moderate-sized omphalocele defect

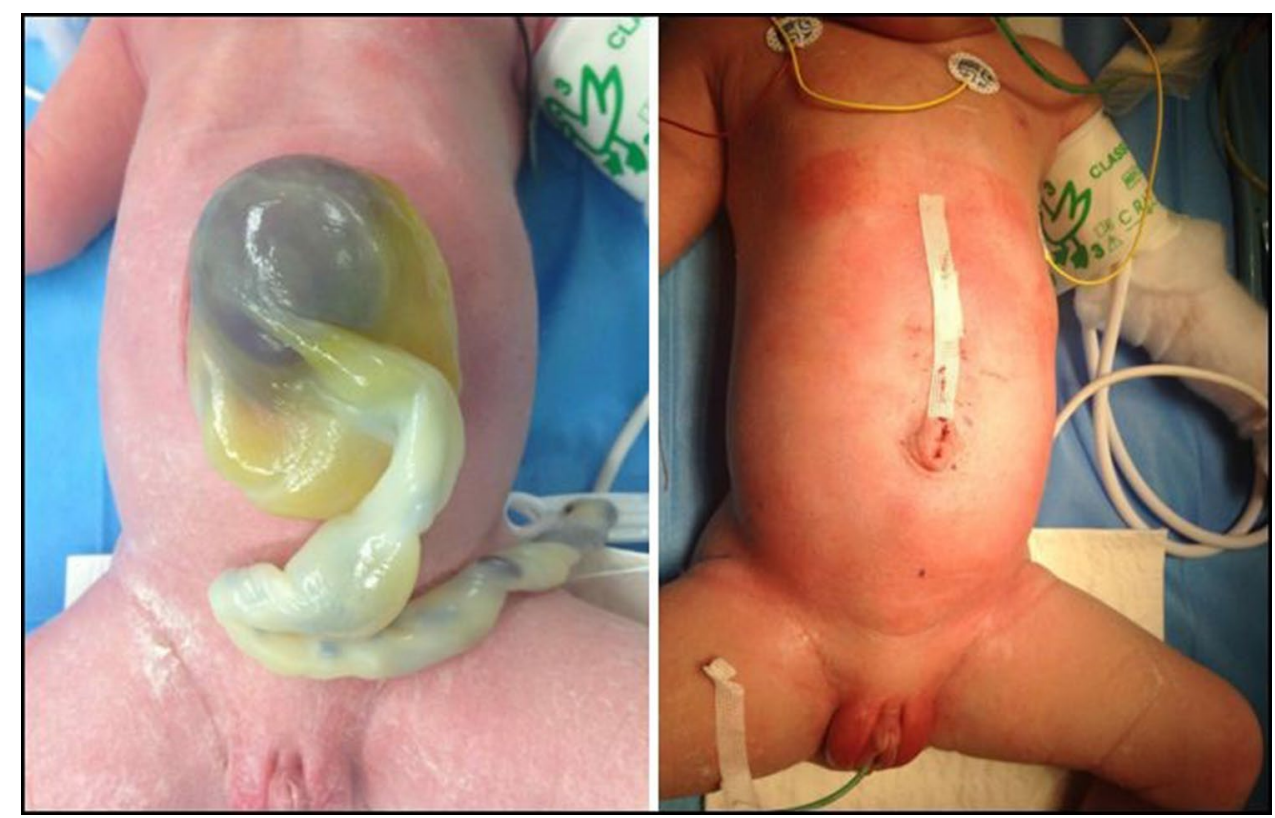




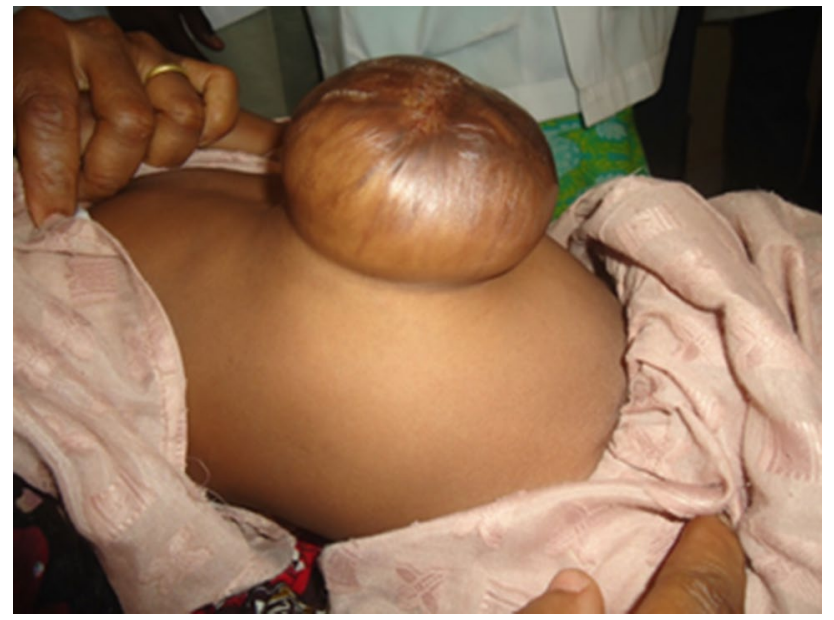

Fig. 6 Ventral hernia formed following non-operative management of omphalocele

Table 7 Selected patient variables (AKTH omphalocele study)

\begin{tabular}{llll}
\hline Parameter & Minimum & Maximum & Median \\
\hline Age of mother in years & 16 & 45 & 26 \\
Parity (no. of births) of mother & 1 & 10 & 4 \\
Age of father in years & 25 & 60 & 40 \\
Weight on admission in kg & 1.5 & 3.9 & 2.58 \\
Length of hospital stay in days & 1 & 47 & 12 \\
\hline
\end{tabular}

\section{Treatment outcome}

The survival in the AKTH series of omphalocele patients was high, owning to the use of non-operative management. Similar findings have been reported from our sub-region [5, $47,53]$. The mortalities in this series were in the patients who had a ruptured omphalocele $(6 / 40 ; 15 \%)$. When the sac ruptures, the infant is exposed to the same challenges as the child with a gastroschisis, with the attendant risk of body fluid and heat losses and the development of septicaemia. Given the size of the fascial defect, the improvised preformed silo cannot be used in these cases, as it cannot be held easily in place. We thus have to employ a sutured silo under general anaesthesia. Due to the size of the defect, and our inability to paralyse and ventilate the patient, reduction is slow, with the wound easily becoming infected and result in the separation of the silo from the fascia and exposing the peritoneal cavity [11]. The attendant sepsis, hypothermia and malnutrition result in a dismal outcome for such babies with a ruptured omphalocele sac [8].
The overall reported survival rate of live-born omphalocele cases is $75-81 \%$ in the current literature. The outcome for omphalocele correlates directly with the size of the defect [54].

Patients with isolated omphalocele have the best 1-year survival, which is greater than $90 \%$, but this is a rather small group of omphalocele cases (around 30\%). Giant omphalocele (defect containing more than $75 \%$ of liver in the sac and/or defect diameter larger than $5 \mathrm{~cm}$ ) have an in-hospital mortality of up to $20 \%$ [55].

Long-term medical problems occur such as gastroesophageal reflux, pulmonary insufficiency, recurrent lung infections or asthma, feeding difficulties, and failure to thrive [55, 56]. Special attention has been focused on the neurodevelopmental outcome of patients with omphalocele. A recent study has shown neurological impairments in more than half of giant omphalocele survivors [57].

\section{HIC lessons learnt and LMIC priorities for omphalocele}

Omphalocele carries a higher survival rate compared to gastroschisis in LMIC. Nevertheless, thorough further research and increased awareness of this congenital anomaly would further improve the outcome. Facilitation of prenatal ultrasound would enable the early diagnosis and referral of these unborn patients to appropriate tertiary centres which will carry out further investigations and planned delivery. As in gastroschisis, out-born cases need to be resuscitated and carefully transported.

Newborns with omphalocele require comprehensive postnatal assessment for accompanying anomalies, especially cardiac anomalies. This requires postnatal ultrasound, echocardiography, and possible X-ray (Fig. 7).

\section{Conclusion}

The current variations in GS and omphalocele outcomes between HIC and LMIC should not be tolerated by the health-care and global community at large. Within LMICs, efforts should be directed at establishing affordable, practical bundles of care, based on the principles outlined above. In addition to rolling out service delivery solutions, clinical, implementation science, and financial implication studies should be carried out to aid impact assessments and strategic planning. It is expected that with these measures along with other generic and specific ones, substantial falls in GS and omphalocele mortality will be demonstrated soon. 
Fig. 7 The AKTH Kano omphalocele algorithm (adapted from Sowande et al. [5])

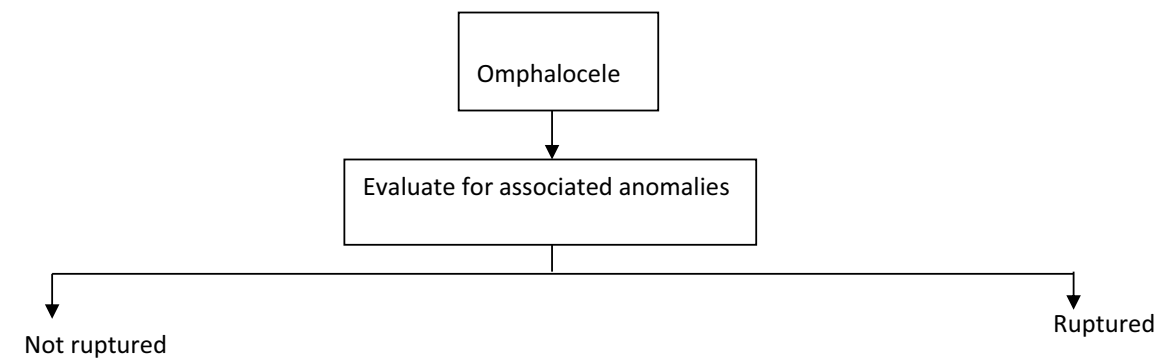

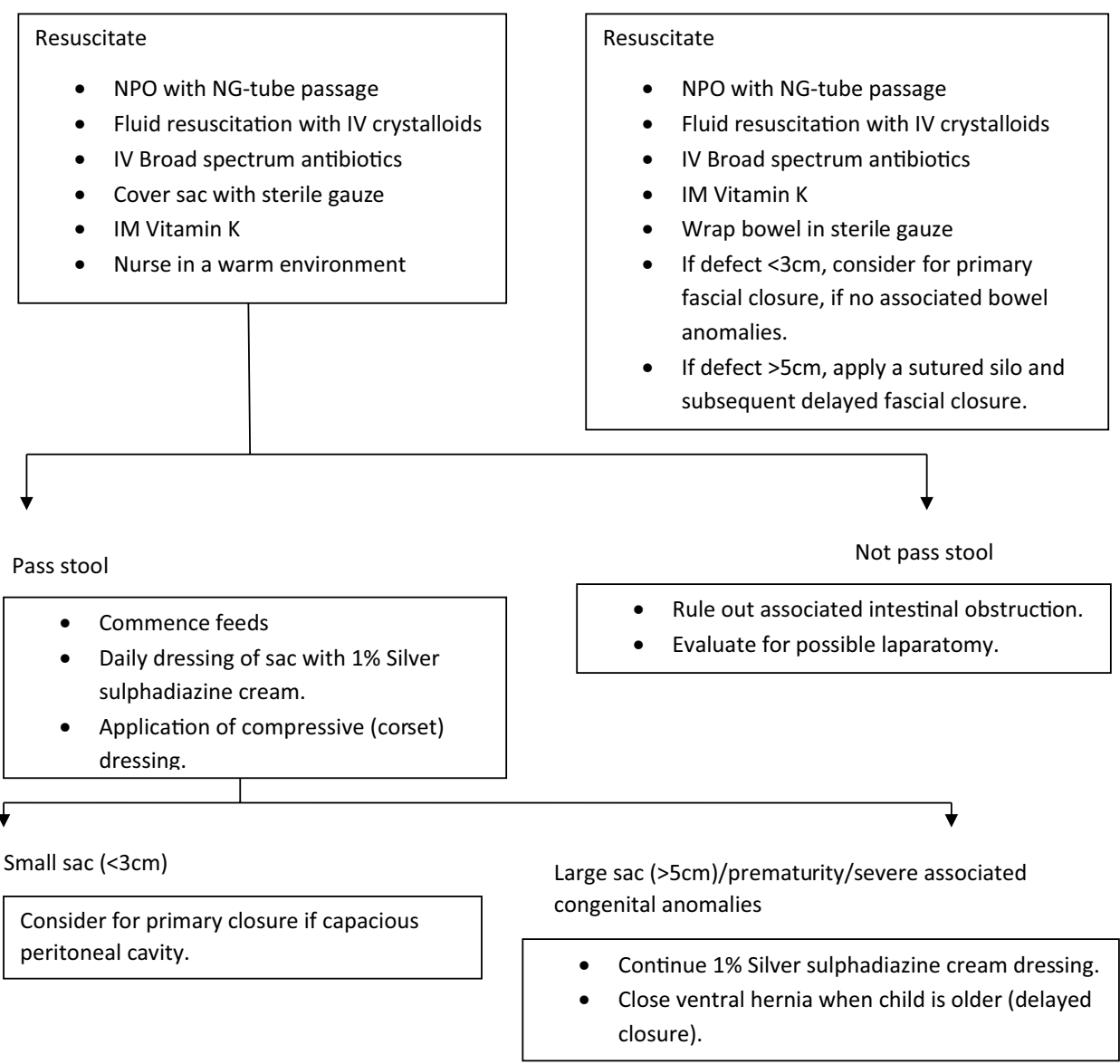

\section{Acknowledgements Open Access funding provided by Projekt DEAL.}

Open Access This article is licensed under a Creative Commons Attribution 4.0 International License, which permits use, sharing, adaptation, distribution and reproduction in any medium or format, as long as you give appropriate credit to the original author(s) and the source, provide a link to the Creative Commons licence, and indicate if changes were made. The images or other third party material in this article are included in the article's Creative Commons licence, unless indicated otherwise in a credit line to the material. If material is not included in the article's Creative Commons licence and your intended use is not permitted by statutory regulation or exceeds the permitted use, you will need to obtain permission directly from the copyright holder. To view a copy of this licence, visit http://creativecommons.org/licenses/by/4.0/.

\section{References}

1. Paul VK, Singh M (2004) Regionalized perinatal care in developing countries. Semin Neonatol 9:117-124

2. Sitkin NA, Ozgediz D, Donkor P et al (2015) Congential anomalies in low- and middle-income countries: the unborn child of global surgery. World J Surg 39:36-40

3. Ameh EA, Seyi-Olajide JO, Sholadoye TT (2015) Neonatal surgical care: a review of the burden, progress and challenges in sub-Saharan Africa. Paediatr Int Child H 35:243-251

4. Ameh EA, Dogo PM, Nmadu PT (2001) Emergency neonatal surgery in a developing country. Pediatr Surg Int 17:448-451

5. Sowande OA, Anyanwu LJC, Inyang AW et al (2013) Syndromic exomphalos in Ile-Ife Nigeria: Management challenges. Arch Int Surg 3:222-225 
6. Wesonga AS, Fitzgerald TN, Kabuye R et al (2016) Gastroschisis in Uganda: opportunities for improved survival. J Pediatr Surg 51:1772-1777

7. Apfeld JC, Wren SM, Macheka $\mathrm{N}$ et al (2015) Infant, maternal and geographic factors influencing gastroschisis related mortality in Zimbabwe. Surgery 158:1475-1480

8. Ameh EA, Chirdan LB (2000) Ruptured exomphalos and gastroschisis: a retrospective analysis of morbidity and mortality in Nigerian children. Pediatr Surg Int 16:23-25

9. Manson J, Ameh E, Canvassar N et al (2012) Gastroschisis: a multi-centre comparison of management and outcome. Afr J Paediatr Surg 9:17-21

10. Ford K, Poenaru D, Moulot O et al (2016) Gastroschisis: Bellwether for neonatal surgery capacity in low resource settings? J Pediatr Surg. https://doi.org/10.1016/j.pedsurg.2016.02.090

11. Ledbetter DJ (2006) Gastroschisis and omphalocele. Surg Clin N Am 86:249-260

12. ElTayeb AA, Helmy AA (2009) Management of Gastroschisis with limited resources. Ann Pediatr Surg 5:166-171

13. Arnold M (2004) Is the incidence of Gastroschisis rising in South Africa in accordance with international trends? A retrospective analysis at Pretoria Academic and Kalafong hospitals, 1981-2001. S Afr J Surg 43:86-88

14. Christison-Lagay ER, Kelleher CM, Langer JC (2011) Neonatal abdominal wall defects. Semin Fetal Neonat Med 16:164-172

15. Kastenberg ZJ, Dutta S (2013) Ventral abdominal wall defects. NeoReviews 14:e402-e411

16. Ali OM, Ali AE (2014) Incidence, clinical presentation and outcome of gastroschisis and omphalocele in Sudan. Sudan Med J 50:75-80

17. Wilson RD, Johnson MP (2004) congenital abdominal wall defects: an update. Fetal Diagn Ther 19:385-398

18. Hibbs SD, Bennett A, Castro Y et al (2016) Abdominal wall defects among Mexican American infants: the effect of maternal nativity. Ethn Dis 26:165-170

19. Jwa E, Kim SC, Kim DY et al (2014) The prognosis of Gastroschisis and omphalocele. J Korean Assoc Pediatr Surg 20:38-42

20. Poddar R, Hartley L (2009) Exomphalos and gastroschisis continuing education in anaesthesia. Crit Care Pain 9:48-51

21. Molik KA, Gingalewski CA, West KW et al (2001) Gastroschisis: a plea for risk categorization. J Pediatr Surg 36:51-55

22. Wright NJ, Zani A, Ade-Ajayi N (2015) Epidemiology management and outcome of gastroschisis in sub Saharan Africa: results of an international survey. Afr J Pediatr Surg 12:1-6

23. Chesley PM, Ledbetter DJ, Meehan JJ et al (2015) Contemporary trends in the use of primary repair for gastroschisis in surgical infants. Am J Surg 209:901-906

24. Risby K, Jakobsen MS, Qvist N (2016) Congenital abdominal wall defects: staged closure by dual mesh. J Neonatal Surg 5:2

25. Allotey J, Davenport M, Nijere I et al (2007) Benefit of preformed silos in the management of gastroschisis. Pediatr Surg Int 23:1065-1069

26. Kunz SN, Tieder JS, Whitlock K et al (2013) Primary fascial closure versus staged closure with silo in patients with gastroschisis. A meta-analysis. J Pediatr Surg 48:845-857

27. Weil BR, Leys CM, Rescorla FJ (2012) The jury is still out: changes in gastroschisis management over the last decade are associated with both benefits and shortcomings. J Pediatr Surg 47:119-124

28. Abdullah F, Arnold MA, Nabaweesi R et al (2007) Gastroschisis in the United States 1988-2003: analysis and risk categorization of 4344 patients. J Perinatol 27:50-55

29. Mendez-Martinez S, Garcia-Carrasco M, Mendozo-Pinto C et al (2016) Surgical complications of simple and complex gastroschisis in newborn. Eur J Gen Med 13:88-93
30. Kuleva M, Khen-Dunlop N, Dumez Y et al (2012) Is complex gastroschisis predictable by prenatal ultrasound? BJOG 119:102-109

31. de Buys Roessingh AS, Damphousse A, Ballabeni P et al (2015) Predictive factors at birth of the severity of gastroschisis. World $\mathbf{J}$ Gastrointest Pathophysiol 6:228-234

32. Naidu RR, Lee FH, Teh KH (1996) Management of gastroschisis in a peripheral hospital setting. Med J Malaysia 51:444-446

33. Covey KM, Hornik CP, Laughon MM et al (2014) Frequency of anomalies and hospital outcomes in infants with gastroschisis and omphalocele. Early Hum Dev 90:421-424

34. Sowande OA, Anyanwu LJC, Talabi AO et al (2010) Pentalogy of Cantrell: a report of three cases. J Surg Tech Case Rep 2:20-23

35. Conner P, Vejde JH, Burgos CM (2018) Accuracy and impact of prenatal diagnosis in infants with Omphalocele. Pediatr Surg Int 38:629-633

36. Springett A, Draper ES, Rankin J et al (2014) Birth prevalence and survival of Exomphalos in England and Wales: 2005 to 2011. Birth Defects Res (Part A) 100:721-725

37. Mortellaro VE, St. Peter SD, Fike FB et al (2011) Review of the evidence on the closure of abdominal wall defects. Pediatr Surg Int 27:391-397

38. Fleurke-Rozema H, van de Kamp K, Bakker M et al (2017) Prevalence, timing of diagnosis and pregnancy outcome of abdominal wall defects after the introduction of a national prenatal screening program. Prenat Diagn 37:383-388

39. Verla MA, Style CC, Olutoye OO (2019) Prenatal diagnosis and management of omphalocele. Semin Pediatr Surg 28:84-88

40. Fawley JA, Peterson EL, Christensen MA et al (2016) Can omphalocele ratio predict postnatal outcomes? J Pediatr Surg 51:62-66

41. Deng K, Qiu J, Dai L et al (2014) Perinatal mortality in pregnancies with omphalocele: data from the Chinese nations birth defects monitoring network, 1996-2006. BMC Pediatr 14:160-167

42. Gamba P, Midrio P (2014) Abdominal wall defects: prenatal diagnosis, newborn management, and long-term outcomes. Semin Pediatr Surg 23:283-290

43. McNair C, Hawes J, Urquhart H (2005) Caring for the newborn with an omphalocele. Neonatal Netw 25:319-327

44. Ayub SS, Taylor JA (2019) Cardiac anomalies associated with omphalocele. Semin Pediatr Surg 28:111-114

45. Gonzalez KW, Chandler NM (2019) Ruptured omphalocele: diagnosis and management. Semin Pediatr Surg 28:101-105

46. Rattan KN, Singh J, Jakhar R et al (2018) Omphalocele: 15 years experience from a single centre in developing country. J Clin Neonatol 7:125-129

47. Ekot EA, Emordi VC, Osifo DO (2017) Does omphalocele major undergo spontaneous closure? J Surg Case Rep 8:1-3

48. Davis AS, Blumenfeld Y, Rubesova E et al (2008) Challenges of giant omphalocele: from, fatal diagnosis to follow up. Neoreviews 9:e338-e347

49. Islam S (2012) Advances in surgery for abdominal wall defects. Clin Perinatol 39:375-386

50. Roux N, Jakubowicz D, Salomon L et al (2018) Early surgical management for giant omphalocele: results and prognostic factors. J Pediatr Surg 53:1908-1913

51. Baumann B, Stephens D, Gershone H et al (2016) Management of giant omphaloceles: a systematic review of methods staged surgical vs. nonoperative delayed closure. J Pediatr Surg 51:1725-1730

52. Mack AJ, Rogdo B (2016) Giant omphalocele: current perspective. Res Rep Neonatal 6:33-39

53. Bode CO, Ademuyiwa AO, Elebute OA (2018) Formal saline versus honey as Escharotic in the conservative management of major omphalocele. Niger Postgrad Med J 25:48-51

54. Raymond SL, Downard CD, St. Peter SD et al (2019) Outcomes in omphalocele correlate with size of defect. J Pediatr Surg $54: 1546-1550$ 
55. Baerg JE, Munoz AN (2019) Long term complications and outcomes in omphaloceles. Semin Pediatr Surg 28:118-121

56. Duggan E, Puligandla P (2019) Respiratory disorders in patients with omphalocele. Semin Pediatr Surg 28:115-117

57. Danzer E, GerdesD'Agostino MJA et al (2015) Patient characteristics are important determinants of neurodevelopmental outcome during infancy in omphalocele. Early Hum Dev 91:187-193
Publisher's Note Springer Nature remains neutral with regard to jurisdictional claims in published maps and institutional affiliations. 